\title{
Superabsorbent hydrogels enhanced by quaternized tunicate cellulose nanocrystals with adjustable strength and swelling ratio
}

\author{
Peng Wei $\cdot$ Weiwei Chen $\cdot$ Qinghua Song $\cdot$ Yibing Wu $\cdot$ Youjia Xu
}

Received: 8 January 2021 / Accepted: 12 February 2021 / Published online: 2 March 2021

(C) The Author(s) 2021

\begin{abstract}
Superabsorbent hydrogels were prepared from biodegradable material, where the matrix was cross-linked cellulose and carboxymethyl cellulose and reinforced by quaternized tunicate cellulose nanocrystals (Q-TCNCs). Due to the large amount of hydrophilic groups on cellulose, carboxymethyl cellulose, and Q-TCNCs, the hydrogels had excellent water absorption capacity. The equilibrium swelling ratio of superabsorbent hydrogels exceeded $500 \mathrm{~g} / \mathrm{g}$ in deionized water and exceeded $120 \mathrm{~g} / \mathrm{g}$ even in synthetic urine, which was higher than those of commercial absorbent materials composed of copolymers of acrylic acid and acrylamide. The water absorption performances and mechanical properties of the hydrogels could be adjusted by changing the content of Q-TCNCs. The resultant materials were expected to replace those polyolefin-based materials, thereby reducing environmental pollution.
\end{abstract}

P. Wei · Y. Xu $(\bowtie)$

Department of Orthopaedics, The Second Affiliated

Hospital of Soochow University, Suzhou 215000,

People's Republic of China

e-mail: xuyoujia2020@163.com;

xuyoujia@suda.edu.com

P. Wei · W. Chen · Q. Song · Y. Wu

Department of Plastic and Reconstructive Surgery,

Ningbo First Hospital, Ningbo 315000, People's Republic

of China
Keywords Superabsorbent · Hydrogels · Cellulose · Carboxymethyl cellulose $\cdot$ Quaternized tunicate cellulose nanocrystals

\section{Introduction}

Superabsorbent hydrogels are hydrophilic networks with high capacities for water uptake, which can absorb, swell, and retain aqueous solutions up to hundreds of times of their own weights (Cipriano et al. 2012; Zhang et al. 2014; Sun et al. 2012). Because hydrogels have three-dimensional polymer network structures, so they can absorb and conserve great amounts of aqueous solutions without dissolving or losing their own structural integrity (Bao et al. 2011; Rashidzadeh and Olad 2014; Rodrigues et al. 2012). The high swelling capacity, good water retention capability, and biodegradability (Kabiri et al. 2003) of superabsorbent hydrogels enable them to have potential applications in various fields, such as agriculture and horticulture (Ibrahim et al. 2010), drug delivery (Gupta et al. 2002), wastewater treatment (Kasgoez and Durmus 2010), self-healing concrete (Mignon et al. 2015), and hygienic products (Kosemund et al. 2009). However, the poor mechanical performances of hydrogels severely hinder their practical applications (Jiang et al. 2017). Therefore, preparation of hydrogels with good mechanical properties is important. In order to solve this problem, different hydrogel network 
structures have been constructed, including double network (DN) hydrogels (Chen et al. 2017), slidingring hydrogels (Bin et al. 2014), macromolecular microsphere composite hydrogels ( $\mathrm{Li}$ et al. 2015), physically cross-linked networks (Chen et al. 2015), and nanocomposite hydrogels (Hu and Chen 2014).

Since the new concept "superabsorbent polymer" that could absorb large amount of water more than hundred times of its own weight appeared in 1950s, the scientific community and industrial world have a strong interest in it, and rapid progress has been made in the past few decades because of the tremendous demand for superabsorbent materials in sanitary industry (Ma et al. 2015). Superabsorbent hydrogels are mainly divided into natural polymeric and synthetic polymeric hydrogels (Gharekhani et al. 2017; Hoffman 2012). Current commercial absorbent materials are mainly polymerized resins that are based on petrochemical products such as acrylic acid, acrylamide, polyvinyl alcohol, and acrylonitrile (Huang et al. 2013). These products are not easy to be degraded. Although the absorbent materials have no direct threat to human life or health, the disposal of synthetic absorbent material waste is a source of various environmental pollutants (Akhter et al. 2004; Nakason et al. 2010). Therefore, natural materials have received great attention. They can be biodegraded, including actions of enzymes, microorganisms and water, transforming into harmless and simple compounds that are environmentally safe. Due to their biodegradability, ease of availability, and non-toxicity compared to synthetic polymers, natural polymers such as chitosan (Zhang et al. 2007), cellulose (Chang et al. 2010), starch (Spagnol et al. 2012), and alginate (Yadav and Rhee 2012) have gained great attention for the preparation of hydrogel materials (Xin et al. 2009).

Cellulose is renewable, biodegradable, and nontoxic. It is the most abundant natural polymer on the earth and is produced in an annual output of $10^{11}-10^{12}$ tons. Cellulose nanocrystals are a substance derived from the crystalline region of cellulose and can be prepared by decomposing, oxidizing, and enzymatically or mechanically separating (Samir et al. 2005; Habibi et al. 2010; Peng et al. 2020; Hu et al. 2020; Klemm et al. 2011; Larssona et al. 2011). Tunicate cellulose nanocrystals are derived from tunicate cellulose, which is an animal fiber derived from tunicate. Compared with other cellulose nanocrystals, tunicate cellulose nanocrystals have higher crystallinity, larger aspect ratio ( $>70)$, and excellent mechanical properties (modulus exceeds $143 \mathrm{GPa}$ ) (Habibi et al. 2010), which have been widely used for the reinforcement of polymeric materials (Peng et al. 2020; Hu et al. 2020). Meanwhile, like other cellulose nanocrystals, tunicate cellulose nanocrystals have a large specific surface area and a large number of reactive functional groups that can be chemically modified. Many cellulose derivatives are readily soluble in water. For example, carboxymethyl cellulose (CMC) is one of the most widely used watersoluble cellulose derivatives in many applications (Sung et al. 2016; Meshram et al. 2009; Gao et al. 2009; Gupta et al. 2007). Chang et al. synthesized superabsorbent hydrogels using CMC and cellulose, whose swelling properties could be controlled by adjusting the ratio of CMC and cellulose (Chang et al. 2010).

In this paper, we used epichlorohydrin (EPI) to crosslink cellulose and CMC chains to form a loose network structure, and positively charged quaternized tunicate cellulose nanocrystalls (Q-TCNCs) were also introduced to form physical crosslinking with CMC chains for adjusting the swelling ratio and strength of hydrogels. Cellulose, CMC and Q-TCNCs contain large amounts of hydroxyl and carboxyl groups, which are beneficial for materials to absorb a large amount of water. Q-TCNCs could not only generate electrostatic interactions with the carboxyl groups of $\mathrm{CMC}$, but also be used as nanofillers to improve the mechanical performance of hydrogel samples. We investigated the effects of electrostatic interactions and nanofillers on the swelling rate and mechanical properties of hydrogels by changing the content of positively charged Q-TCNCs and adding the uncharged H-TCNCs. The swelling ratios of hydrogels in deionized water, simulated physiological fluids $(0.5 \mathrm{wt} \%$ glucose solution, $0.5 \mathrm{wt} \%$ urea solution, $0.9 \mathrm{wt} \%$ physiological saline, synthetic urine solution), and salt solutions (such as $\mathrm{NaCl}, \mathrm{CaCl}_{2}$ and $\mathrm{FeCl}_{3}$ solutions) were tested. The mechanical properties of hydrogels were also tested after they reached equilibrium swelling in deionized water. This water-absorbing material composed of renewable materials is expected to be a substitute for synthetic water-absorbing materials, thereby reducing fossil energy consumption and environmental pollution. 


\section{Experimental}

\section{Materials}

Tunicate (Halocynthiaroretzi Drasche) was purchased from Weihai Evergreen Marine science and technology Co. Ltd (Shandong, China). Carboxymethyl cellulose $\left(\mathrm{CMC}, 2.43 \times 10^{4}\right)$ was analytical grade and purchased from Shanghai Chemical Agents Co. Ltd. The degree of carboxymethyl substitution (DS) is 0.7. Epichlorohydrin (EPI) was analytical grade, and was used without further purification. (2,3-epoxypropyl) trimethylammonium chloride (EPTAC) was purchased from Shanghai Dibo Chemical Technology Co. Ltd. Cellulose cotton linter pulps were provided by Hubei Chemical Fiber Group Ltd. (Xiangyang, China), and viscosity average molecular weight $(\mathrm{M \eta})$ was $9 \times 10^{4}$. Other chemicals were all analytical grade and were used without further purification. All solutions were prepared with deionized water.

\section{Preparation of Q-TCNCs}

Tunicate cellulose was first isolated from the mantles of sessile sea creatures known as tunicates, and then hydrolyzed with hydrochloric acid to obtain tunicate cellulose nanocrystals (H-TCNCs) (Zhang et al. 2017). $7.5 \mathrm{~g}$ dried tunicate cellulose was dispersed in $300 \mathrm{~mL}$ hydrochloride acid (4 M), and stirred at a speed of $300 \mathrm{rpm}$ at $100{ }^{\circ} \mathrm{C}$ for reflux condensation $(4 \mathrm{~h})$. The obtained solutions were diluted with distilled water after removal of large particles and repeated centrifugation under $7000 \mathrm{rpm}$ until neutrality. The TCNC suspensions were obtained and their concentrations were measured by gravimetric method.

Sodium hydroxide $(7 \mathrm{wt} \%)$ was added into $\mathrm{H}-\mathrm{TCNC}$ suspension as catalyst of etherification. EPTAC (10 equiv/AGU groups) was slowly dropped into suspension with stirring at $65{ }^{\circ} \mathrm{C}$ and reacted for $24 \mathrm{~h}$. The final products were dialyzed (molecular weight cut off $=8000 \mathrm{Da}$ ) against distilled water for 4 days. After reverse dialysis against high concentrated PVA, the concentration of Q-TCNCs suspension was about $2.3 \%$.

Preparation of superabsorbent hydrogels

Cellulose cotton linter (3 wt\%) was dissolved with 7 $\mathrm{wt} \% \mathrm{NaOH} / 12 \mathrm{wt} \%$ urea solution (Chang et al. 2010).
Then, Q-TCNCs and CMC were added to $7 \mathrm{wt} \%$ $\mathrm{NaOH} / 12 \mathrm{wt} \%$ urea solutions in different proportions, and the total concentration of CMC and Q-TCNCs was $3 \mathrm{wt} \%$ (as shown in Table 1). The above two solutions were mixed at a mass ratio of 1:9 (cellulose solution/ CMC and Q-TCNCs solution), and stirred in an ice bath for $0.5 \mathrm{~h}$. After EPI was added into the mixture under stirring, the mixture was poured into a mold and placed in a $60{ }^{\circ} \mathrm{C}$ oven for $12 \mathrm{~h}$. Finally, the gels were taken out and washed sufficiently with distilled water to remove $\mathrm{NaOH}$, urea, and residual EPI. The obtained hydrogel samples were coded as Q-0, Q-4, Q-10, and $\mathrm{H}-10$, respectively, according to the proportion of the components. The preparation process is shown in Fig. 1.

\section{Characterization}

Transmission electron microscope (TEM) observation of TCNCs was performed by using a JEM-2010 transmission electron microscope (JEOL, Japan) with an acceleration voltage of $200 \mathrm{kV}$. A droplet of dilute TCNC suspension $(0.01 \mathrm{wt} \%)$ was dropped and dried on a copper grid before measurement. Fourier transfer infrared spectroscopy (FTIR) was carried out by NICOLET 5700 FTIR spectrometer (Thermo Fisher Scientific, USA). The samples were prepared by using the $\mathrm{KBr}$ method and completely dried under an infrared lamp before testing. Zeta potential was measured by Zeta Sizer Nano series Nano-ZS (Malvern Instruments Ltd, Malvern, UK) at room temperature. The mechanical properties of hydrogels were tested at room temperature by using a universal testing machine (CMT6350, Shenzhen SANS, China). For the compression test, the dimension of swollen samples was about $25 \mathrm{~mm}(\mathrm{D}) \times 16 \mathrm{~mm}(\mathrm{H})$. The crosshead speed was $0.5 \mathrm{~mm} / \mathrm{min}$, and the initial height of the hydrogel samples for compressive test between two clamps was $16 \mathrm{~mm}$. The compression test of each sample was repeated for five times.

The equilibrium swelling ratio (ESR) of hydrogels was measured by gravimetric method. The ESR value was calculated as follows:

$\mathrm{ESR}=\frac{W_{\mathrm{e}}-W_{\mathrm{d}}}{W_{\mathrm{d}}}$

where $W_{\mathrm{e}}$ was the weight of swollen hydrogels at equilibrium and $W_{\mathrm{d}}$ was the weight of dried gel. 
Table 1 Reaction conditions and components for superabsorbent hydrogels

\begin{tabular}{lllllll}
\hline Samples & TCNCs $(\mathrm{wt} \%)$ & $\mathrm{CMC}(\mathrm{wt} \%)$ & Cellulose $(\mathrm{wt} \%)$ & $\mathrm{EPI}(\mathrm{mL} / \mathrm{g})$ & Time $(\mathrm{h})$ & Temperature $\left({ }^{\circ} \mathrm{C}\right)$ \\
\hline Q-0 & 0 & 3 & 3 & 0.1 & 12 & 60 \\
Q-4 & 0.12 & 2.88 & 3 & 0.1 & 12 & 60 \\
Q-10 & 0.3 & 2.7 & 3 & 0.1 & 12 & 60 \\
H-10 & 0.3 & 2.7 & 3 & 0.1 & 12 & 60 \\
\hline
\end{tabular}

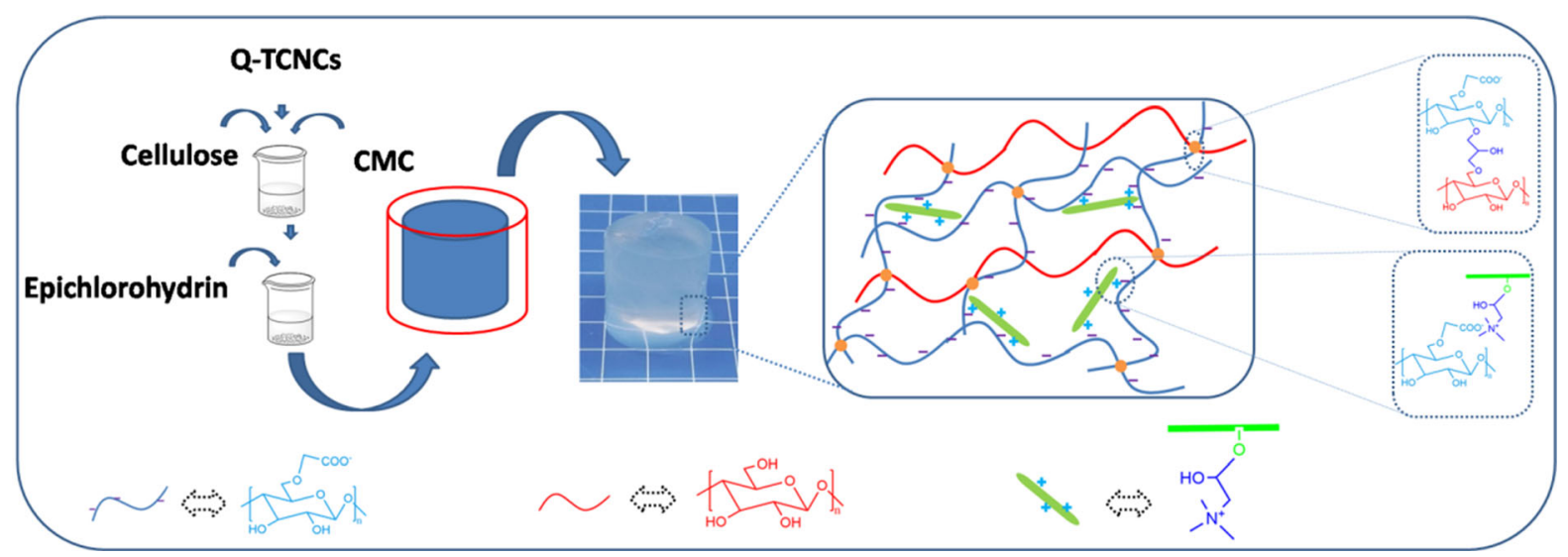

Fig. 1 Preparation process and structure of superabsorbent hydrogel

The effective crosslink density $\left(v_{\mathrm{c}}{ }^{*}\right)$ of hydrogels was calculated based on an affine network model as follows (Tang et al. 2011):

$v_{\mathrm{c}}^{*}=G /\left(R T v_{2, \mathrm{r}}^{2 / 3} v_{2, \mathrm{~s}}^{1 / 3}\right)$

where $G$ was shear modulus of hydrogels reaches equilibrium swelling, $R$ was general gas constant, $T$ was Kelvin temperature (K), $v_{2, \mathrm{r}}$ and $v_{2, \mathrm{~s}}$ were volume fraction of relaxation state of the polymer after completion of the polymerization reaction and swelling equilibrium respectively. Equations are as follows:

$v_{2, \mathrm{r}}=M_{\mathrm{r}} C_{0} / \rho_{\mathrm{p}}$

$v_{2, \mathrm{~s}}=\left[\left(1+\operatorname{ESR} \rho_{\mathrm{p}}\right) / \rho_{\mathrm{s}}\right]^{-1}$

where $M_{\mathrm{r}}$ was average molecular weight of polymer repeating units, $C_{0}$ was total monomer concentration in the polymer, $\rho_{\mathrm{p}}$ and $\rho_{\mathrm{s}}$ were density of hydrogels and solvent respectively. $\rho_{\mathrm{p}}$ was calculated as follows:

$\rho_{\mathrm{p}}=m_{\mathrm{p}} / V_{\mathrm{p}}$ where $m_{\mathrm{p}}$ and $V_{\mathrm{p}}$ were mass and volume of dry gel respectively.

\section{Results and discussion}

Construction of superabsorbent hydrogels

In order to develop a superabsorbent hydrogel with considerable mechanical performance, we designed a superabsorbent hydrogel with adjustable mechanical properties and swelling property in this work. As shown in Fig. 1, CMC and cellulose were chemically cross-linked by EPI, and hydrogel networks were also reinforced by positively charged Q-TCNCs. Hydrogels composed of such network structures could absorb large amounts of water due to a large number of hydrophilic groups such as carboxyl groups and hydroxyl groups on CMC, cellulose, and Q-TCNCs. The hydrogel had hybrid cross-linked network structures, where cellulose and CMC were chemically cross-linked by EPI and the negatively charged CMC chains were physically cross-linked by positively 
charged Q-TCNCs through the electrostatic interaction. Furthermore, Q-TCNCs acted as nanofillers in the polymeric matrix.

In order to bring positive charges to the surface of uncharged H-TCNCs, EPTAC was used as the etherification reagent. Through the etherification reaction of hydroxyl groups on the surface of H-TCNCs with EPTAC, the surfaces of H-TCNCs were modified with quaternary ammonium groups to obtain positively charged Q-TCNCs. The effect of the amount of EPTAC on the surface charge of Q-TCNCs was investigated. As shown in Fig. 2, the abscissa was the molar ratios of EPTAC to hydroxyl groups on the surface of H-TCNCs, and the ordinate was the zeta potential of Q-TCNCs. The zeta potential of $\mathrm{H}$-TCNCs was $0 \mathrm{mV}$, which proved that the surface of H-TCNCs was uncharged. As the content of EPTAC increased, the zeta potential of Q-TCNCs gradually increased. Finally, when the EPTAC/-OH exceeded 5:1, the zeta potential of Q-TCNCs was maintained above $+52 \mathrm{mV}$ with no significant increase. Therefore, we chose EPTAC/-OH of 5:1 for the etherification reaction to maximize the positive charge density on the surface, thereby obtaining a stronger electrostatic interaction with the negatively charged carboxyl groups on CMC.

The morphology of TCNCs before and after modification was observed with TEM (as shown in Fig. 3). It could be observed in Fig. 3a that some of the uncharged $\mathrm{H}$-TCNCs aggregated and formed bundle structures, while the nanocrystals modified by

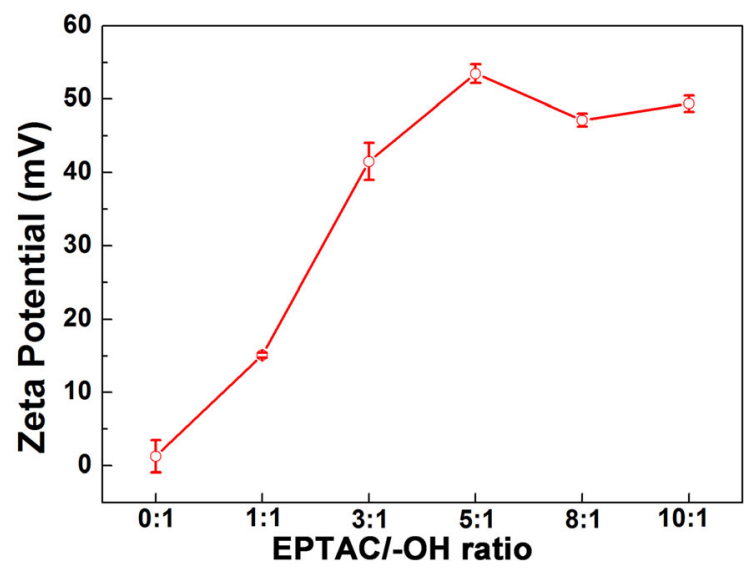

Fig. 2 Zeta potential of quaternized tunicate cellulose nanocrystals varies with the molar ratio of quaternary ammonium reagent to hydroxyl group positively charged quaternary ammonium groups showed better dispersion (Fig. 3b). Due to the modification with quaternary ammonium groups (etherification reaction of EPTAC/-OH was 5:1), the surface of Q-TCNCs was positively charged (Zeta potential exceeded $+52 \mathrm{mV}$ ), so the electrostatic repulsion occurred for better dispersion. At the same time, it was found that the cellulose nanocrystals still maintained rod-like shape, which proved that the etherification reaction did not affected the morphology of cellulose nanocrystals.

Figure 4 shows FTIR spectra of Q-10, CMC, cellulose, and Q-TCNCs. It can be seen that the broad peak of $3300-3500 \mathrm{~cm}^{-1}$ was attributed to the stretching vibration peak of $-\mathrm{OH}$, while the peaks at $2930 \mathrm{~cm}^{-1}$ and $2860 \mathrm{~cm}^{-1}$ could be assigned to the symmetric and asymmetric stretching vibrations of $\mathrm{CH}_{2}$ groups. The characteristic peaks located at $1600 \mathrm{~cm}^{-1}$ and $1420 \mathrm{~cm}^{-1}$ were the stretching vibration and bending vibration of $-\mathrm{COOH}$ groups. It could be observed that Q-10 had cellulose characteristic peaks of $1450 \mathrm{~cm}^{-1}, 1315 \mathrm{~cm}^{-1}, \quad 1150 \mathrm{~cm}^{-1}$, $1050 \mathrm{~cm}^{-1}$ and $1020 \mathrm{~cm}^{-1}$ and characteristic peaks of carboxyl groups at $1600 \mathrm{~cm}^{-1}$ and $1420 \mathrm{~cm}^{-1}$, proving that cellulose and CMC were cross-linked in hydrogel networks.

Swelling properties of superabsorbent hydrogels

The swelling properties of hydrogels in different solutions were investigated. As shown in Fig. 5, all the samples had the same size after removing from molds. However, the sizes of hydrogel samples changed after reaching swelling equilibrium, where Q-0 had the largest size and Q-10 was the smallest sample, suggesting that Q-0 absorbed the most water, and Q-10 absorbed the least water. After reaching the swelling equilibrium, the hydrogels could still maintain their shapes without being damaged. At the same time, the hydrogels gradually became opaque as the content of cellulose nanocrystals increased, because cellulose nanocrystals could hinder the propagation of light after aggregation. As shown in Fig. 5b, after the samples were dried, their volumes decreased a lot, which also indicated the high water contents in the hydrogels.

The water absorption capacity was tested at equilibrium swelling of the samples in a variety of solutions $(0.5 \mathrm{wt} \%$ D-glucose solution, $0.5 \mathrm{wt} \%$ urea 
a

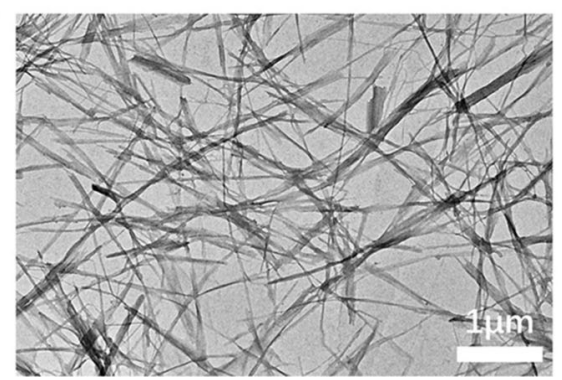

Fig. 3 TEM images of H-TCNCs (a) and Q-TCNCs (b)

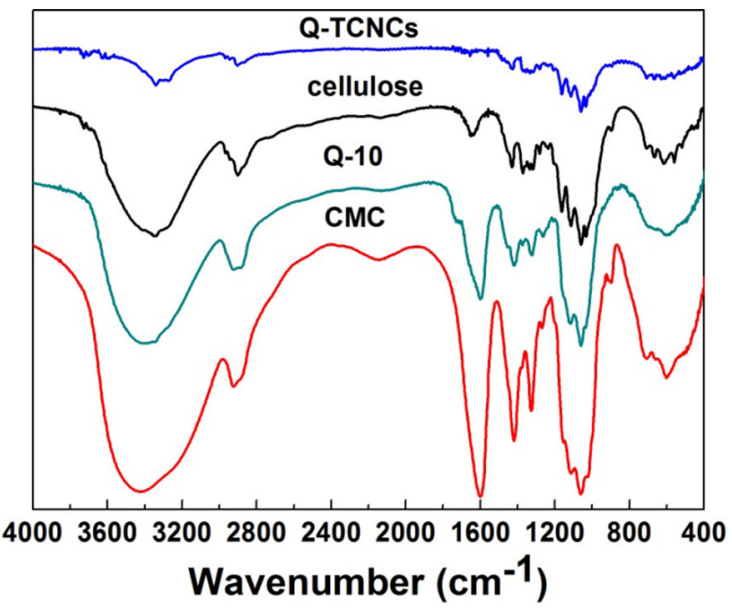

Fig. 4 FTIR spectra of Q-TCNCs, cellulose, Q-10, and CMC

solution, $0.9 \mathrm{wt} \%$ physiological saline concentration, and the synthetic urine containing $0.8 \mathrm{wt} \% \mathrm{NaCl}, 0.1$ wt $\% \mathrm{MgSO}_{4}, 2$ wt $\%$ urea, and 0.02 wt $\% \mathrm{CaCl}_{2}$ ). Figure 6 shows the equilibrium swelling ratio of each sample in various solutions. After each sample reached swelling equilibrium in deionized water, 0.5

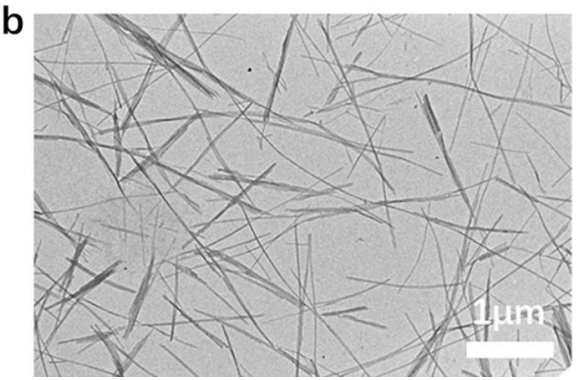

wt $\%$ glucose solution, and $0.5 \mathrm{wt} \%$ urea solution, their equilibrium swelling ratios did not change much, because both the glucose solution and the urea solution are non-ionic solutions. However, after reaching the swelling equilibrium in physiological saline and synthetic urine, their equilibrium swelling ratios decreased from 500 to $756 \mathrm{~g} / \mathrm{g}$ in deionized water to 150-200 g/g in physiological saline and synthetic urine. Because there were $\mathrm{Na}^{+}$and $\mathrm{Cl}^{-}$in physiological saline, and other ions such as $\mathrm{Ca}^{2+}, \mathrm{Mg}^{2+}, \mathrm{SO}_{4}{ }^{2-}$ in the synthetic urine, which electrostatically shielded the carboxylate groups on CMC. The electrostatic repulsion of hydrogel networks was weakened, thereby reducing the expansion between the molecular chains, and finally lowering the equilibrium swelling ratios. The equilibrium swelling ratios of different samples were also compared. For example, the swelling ratio of Q-0 reached $756 \mathrm{~g} / \mathrm{g}$ in deionized water, but gradually decreased with the increase of Q-TCNC content. The equilibrium swelling ratios of Q-4, Q-10, and H-10 were $713 \mathrm{~g} / \mathrm{g}, 516 \mathrm{~g} / \mathrm{g}$, and $605 \mathrm{~g} / \mathrm{g}$, respectively. It could be concluded that as the

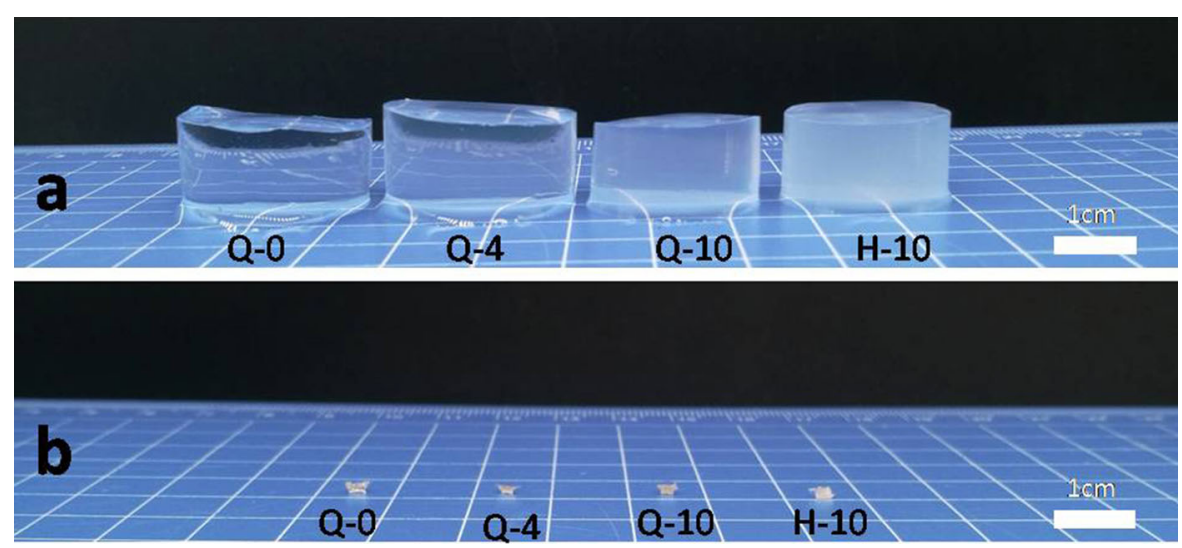

Fig. 5 Photographs of superabsorbent hydrogels: swollen hydrogels (a) and dried hydrogels (b) 


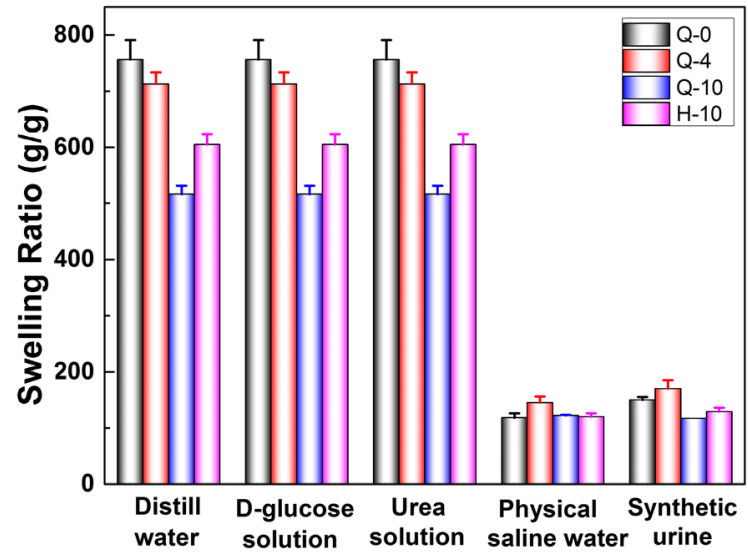

Fig. 6 Equilibrium swelling ratio of Q-0, Q-4, Q-10, and H-10 in different simulated physiological fluids

electrostatic interaction increased, the network structure of the hydrogels became more compact, thereby reducing the swelling ratios. By comparing the swelling ratio of Q- 0 and $\mathrm{H}-10$, it can be seen that the effect of the nanofillers also enhanced the interaction of the polymer chains in the network, thereby reducing the swelling ratios. Equilibrium swelling ratios of dried samples after re-swelling were tested and compared to the commercial absorbent material in the diaper (Fig. 7). The equilibrium swelling ratios of Q-0, Q-4, Q-10, and H-10 were $185 \mathrm{~g} / \mathrm{g}, 135 \mathrm{~g} / \mathrm{g}$, $125 \mathrm{~g} / \mathrm{g}$, and $134 \mathrm{~g} / \mathrm{g}$, respectively, but the equilibrium swelling ratio of commercial material was $243 \mathrm{~g} / \mathrm{g}$, which was higher than our samples. However, the opposite results are shown in synthetic urine, where the equilibrium swelling ratio of commercial material

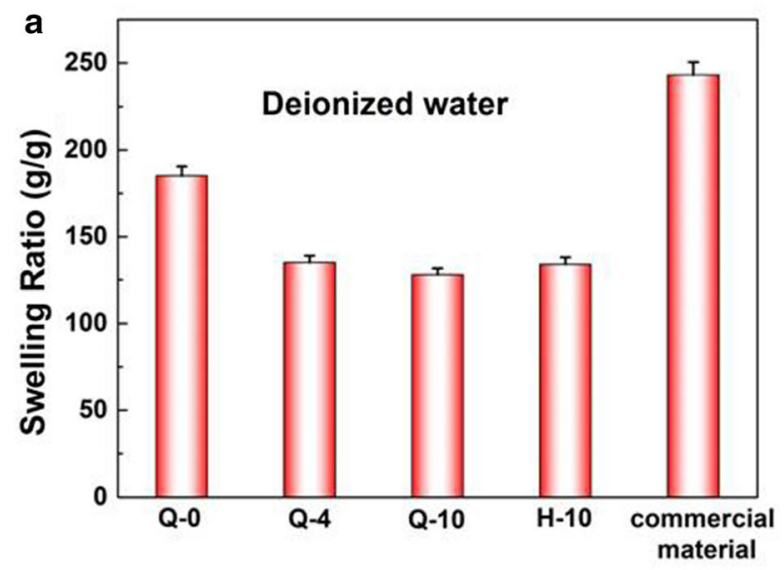

was $53 \mathrm{~g} / \mathrm{g}$, but those of Q-0, Q-4, Q-10 and H-10 were $123 \mathrm{~g} / \mathrm{g}, 97 \mathrm{~g} / \mathrm{g}, 79 \mathrm{~g} / \mathrm{g}$ and $86 \mathrm{~g} / \mathrm{g}$, respectively.

The effects of different concentrations and types of salt solutions on the equilibrium swelling ratios of hydrogels are shown in Fig. 8. The equilibrium swelling ratios of Q-0, Q-4, Q-10, and H-10 gradually reduced with the increase of $\mathrm{NaCl}$ solution concentration. When the concentration of $\mathrm{NaCl}$ exceeded $0.024 \mathrm{~g} / \mathrm{g}$, the concentration of salt solution had little effect on the equilibrium swelling ratios of hydrogels. The equilibrium swelling ratio of Q-0 was largest, and the swelling ratio of Q-10 was smallest in $\mathrm{NaCl}$ solution. They had the same reason as those in physiological saline and synthetic urine. When the carboxylate groups were completely surrounded by positive ions and the electrostatic shielding was saturated, so the equilibrium swelling ratios of hydrogels changed slightly with the increase of ion concentration in the salt solution. As shown in Fig. 8b, the hydrogels had the same trend in the $\mathrm{CaCl}_{2}$ solution. However, there was an opposite trend in $\mathrm{FeCl}_{3}$ solution in Fig. 8c. It might be because of that $\mathrm{Fe}^{3+}$ was trivalent and could cross-link the carboxyl groups on CMC, so the swelling ratio dropped more. In Fig. 8d, the equilibrium swelling ratios of hydrogels in high-valence salt solution decreased faster with the increase of salt concentration, and reduced more after stabilizing, indicating that high-valence salt solution had stronger electrostatic shielding on the carboxyl groups.

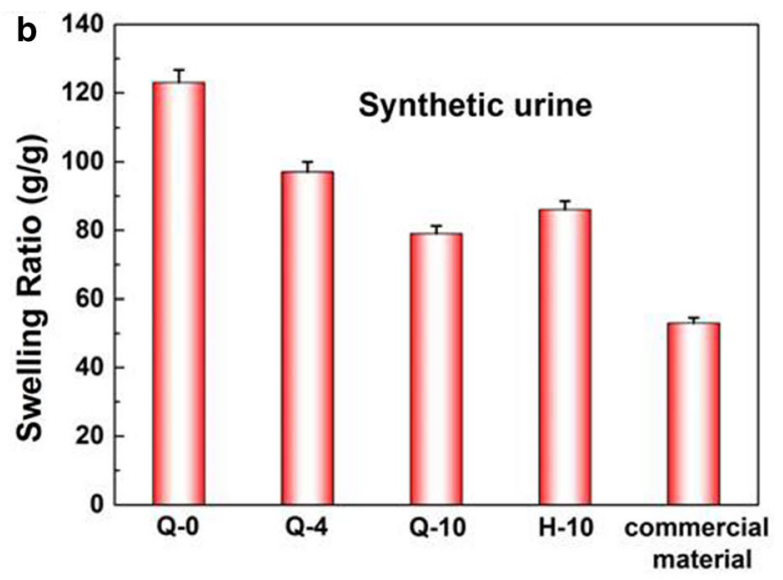

Fig. 7 Equilibrium swelling ratios of each dried sample and commercial absorbing material after re-swelling in deionized water (a) and synthetic urine (b) 

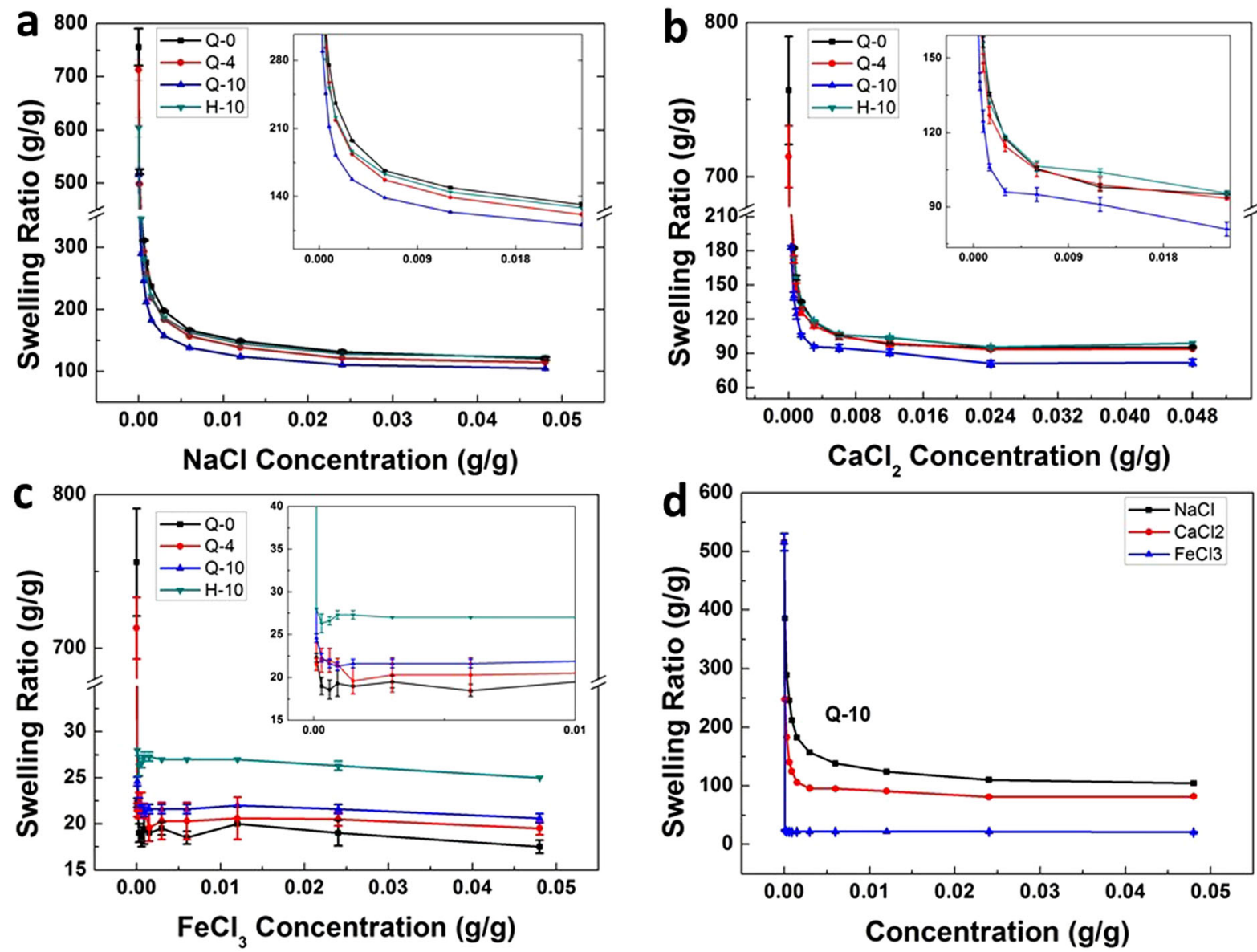

Fig. 8 Effects of different salt solutions on the equilibrium swelling ratios of superabsorbent hydrogels

Mechanical properties and crosslink density of hydrogels

The mechanical properties of hydrogels were tested, where the compressive strength were $1.9 \mathrm{kPa}$, $2.6 \mathrm{kPa}$, and $5.4 \mathrm{kPa}$ for Q-0, Q-4, and Q-10, respectively. As the electrostatic interaction increased, the strength of the hydrogels gradually increased. The compressive strength of Q-0 and $\mathrm{H}-10$ were $1.9 \mathrm{kPa}$ and $2.7 \mathrm{kPa}$, respectively, indicating that the nanofillers were contributed to the strength of hydrogels. From Table 2, it was observed that the effective crosslink density $\left(v_{\mathrm{c}}{ }^{*}\right)$ gradually increased as the content of Q-TCNCs increased. These results were consistent with the mechanical properties and swelling ratio change of the hydrogels.

Table 2 Physical parameters of superabsorbent hydrogels

\begin{tabular}{llllllll}
\hline Samples & ESR $(\mathrm{g} / \mathrm{g})$ & $\rho_{\mathrm{p}}\left(\mathrm{g} / \mathrm{cm}^{3}\right)$ & $v_{2 \mathrm{~s}}$ & $v_{2 \mathrm{r}}$ & $\mathrm{G}(\mathrm{kPa})$ & $v^{*} \mathrm{c}(\mathrm{mol} / \mathrm{L})$ & $\begin{array}{l}\text { Compressive } \\
\text { strength }(\mathrm{kPa})\end{array}$ \\
\hline Q-0 & 756 & 0.32 & 0.0041 & 0.094 & 10 & 0.122 & 1.9 \\
Q-4 & 713 & 0.51 & 0.0027 & 0.057 & 10 & 0.195 & 2.6 \\
Q-10 & 516 & 0.62 & 0.0031 & 0.044 & 20 & 0.443 & 5.4 \\
H-10 & 605 & 0.46 & 0.0035 & 0.059 & 10 & 0.173 & 2.7 \\
\hline
\end{tabular}




\section{Conclusion}

In summary, we synthesized a superabsorbent hydrogel where cellulose and CMC were cross-linked with EPI, and Q-TCNCs as nanofillers were incorporated to form physical cross-linking structures. The equilibrium swelling ratios of the hydrogels exceeded $500 \mathrm{~g} /$ $\mathrm{g}$ in deionized water, and exceeded $120 \mathrm{~g} / \mathrm{g}$ even in synthetic urine. The swelling ratios and mechanical properties of hydrogels could be controlled by adjusting the content of Q-TCNCs. Equilibrium swelling ratios could be adjusted from 512 to $756 \mathrm{~g} / \mathrm{g}$ and mechanical properties could be adjusted from 1.9 to $5.4 \mathrm{kPa}$. This work provided a way for preparation of environmentally friendly materials from natural resources.

Acknowledgments This work was financially supported by Zhejiang Medical Science and Technology Project (Grant No. 2019KY569), and the Natural Foundation of Ningbo (Grant No. 2017A610215).

Open Access This article is licensed under a Creative Commons Attribution 4.0 International License, which permits use, sharing, adaptation, distribution and reproduction in any medium or format, as long as you give appropriate credit to the original author(s) and the source, provide a link to the Creative Commons licence, and indicate if changes were made. The images or other third party material in this article are included in the article's Creative Commons licence, unless indicated otherwise in a credit line to the material. If material is not included in the article's Creative Commons licence and your intended use is not permitted by statutory regulation or exceeds the permitted use, you will need to obtain permission directly from the copyright holder. To view a copy of this licence, visit http://creativecommons.org/licenses/by/4.0/.

\section{References}

Akhter J, Mahmood K, Malik KA et al (2004) Effects of hydrogels amendment on water storage of sandy loam and loam soils and seedling growth of barley, wheat and chickpea. Plant Soil Environ 50(10):463-469

Bao Y, Ma J, Li N (2011) Synthesis and swelling behaviors of sodium carboxymethyl cellulose-g-poly(AA-co-AM-coAMPS)/MMT superabsorbent hydrogels. Carbohydr Polym 84(1):76-82

Bin AI, Esaki K, Gotoh $\mathrm{H}$ et al (2014) Extremely stretchable thermosensitive hydrogels by introducing slide-ring polyrotaxane cross-linkers and ionic groups into the polymer network. Nat Commun 5:5124
Chang C, Duan B, Cai J et al (2010) Superabsorbent hydrogelss based on cellulose for smart swelling and controllable delivery. Eur Polym J 46(1):92-100

Chen Q, Zhu L, Chen H et al (2015) A novel design strategy for fully physically linked double network hydrogelss with tough, fatigue resistant, and self-healing properties. Adv Funct Mater 25(10):1598-1607

Chen H, Liu Y, Ren B et al (2017) Super bulk and interfacial toughness of physically crosslinked double-network hydrogels. Adv Funct Mater 27(44):1703086

Cipriano BH, Banik SJ, Sharma R et al (2012) Superabsorbent hydrogels that are robust and highly stretchable. Macromolecules 47(13):4445-4452

Gao C, Liu M, Chen S et al (2009) Preparation of oxidized sodium alginate-graft-poly((2-dimethylamino) ethyl methacrylate) gel beads and in vitro controlled release behavior of BSA. Int J Pharm 371(1):16-24

Gharekhani H, Olad A, Mirmohseni A et al (2017) Superabsorbent hydrogels made of NaAlg-g-poly(AA-co-AAm) and rice husk ash: synthesis, characterization, and swelling kinetic studies. Carbohydr Polym 168:1-13

Gupta P, Vermani K, Garg S (2002) Hydrogels: from controlled release to $\mathrm{pH}$-responsive drug delivery. Drug Discov Today 7(10):569-579

Gupta KC, Sahoo S, Khandekar K (2007) Graft copolymerization on to cellulose using binary mixture of monomers. J Macromol Sci Part A Chem 44(7):707-719

Habibi Y, Lucia LA, Rojas OJ (2010) Cellulose nanocrystals: chemistry, self-assembly, and applications. Chem Rev 110(6):3479-3500

Hoffman AS (2012) Hydrogels for biomedical applications. Ann N Y Acad Sci 64(1):18-23

Hu Z, Chen G (2014) Novel nanocomposite hydrogels consisting of layered double hydroxide with ultrahigh tensibility and hierarchical porous structure at low inorganic content. Adv Mater 26(34):5950-5956

Hu D, Cui Y, Mo K, Wang J, Huang Y, Miao X, Lin J, Chang C (2020) Ultrahigh strength nanocomposite hydrogels designed by locking oriented tunicate cellulose nanocrystals in polymeric networks. Compos Part B Eng 197:108118

Huang Z, Liu S, Fang G, Zhang B (2013) Synthesis and swelling properties of $\beta$-cyclodextrin-based superabsorbent resin with network structure. Carbohydr Polym 92(2):2314-2320

Ibrahim SM, Salmawi KME, Zahran AH (2010) Synthesis of crosslinked superabsorbent carboxymethyl cellulose/acrylamide hydrogels through electron-beam irradiation. J Appl Polym Sci 104(3):2003-2008

Jiang H, Zhang G, Feng X et al (2017) Room-temperature selfhealing tough nanocomposite hydrogels crosslinked by zirconium hydroxide nanoparticles. Compos Sci Technol 140:54-62

Kabiri K, Omidian H, Hashemi SA et al (2003) Synthesis of fastswelling superabsorbent hydrogels: effect of crosslinker type and concentration on porosity and absorption rate. Eur Polym J 39(7):1341-1348

Kasgoez H, Durmus A (2010) Dye removal by a novel hydrogels-clay nanocomposite with enhanced swelling properties. Polym Adv Technol 19(7):838-845 
Klemm D, Kramer F, Moritz S et al (2011) Nanocelluloses: a new family of nature-based materials. Angew Chem Int Ed 50(24):5438-5466

Kosemund K, Schlatter H, Ochsenhirt JL et al (2009) Safety evaluation of superabsorbent baby diapers. Regul Toxicol Pharmacol 53(2):81-89

Larssona M, Zhoub Q, Larssona A (2011) Different types of microfibrillated cellulose as filler materials in polysodium acrylate superabsorbents. Chin J Polym Sci 29(4):407-413

Li X, Ji G, Zhang H (2015) Phase transitions of macromolecular microsphere composite hydrogels based on the stochastic Cahn-Hilliard equation. J Comput Phys 283:81-97

Ma J, Li X, Bao Y (2015) Advances in cellulose-based superabsorbent hydrogels. RSC Adv 5:59745-59757

Meshram MW, Patil VV, Mhaske ST et al (2009) Graft copolymers of starch and its application in textiles. Carbohydr Polym 75(1):71-78

Mignon A, Graulus GJ, Snoeck D et al (2015) pH-sensitive superabsorbent polymers: a potential candidate material for self-healing concrete. J Mater Sci 50(2):970-979

Nakason C, Wohmang T, Kaesaman A et al (2010) Preparation of cassava starch-graft-polyacrylamide superabsorbents and associated composites by reactive blending. Carbohydr Polym 81(2):348-357

Peng N, Huang D, Gong C, Wang Y, Zhou J, Chang C (2020) Controlled arrangement of nanocellulose in polymeric matrix: from reinforcement to functionality. ACS Nano 14(12):16169-16179

Rashidzadeh A, Olad A (2014) Slow-released NPK fertilizer encapsulated by NaAlg-g-poly(AA-co-AAm)/MMT superabsorbent nanocomposite. Carbohydr Polym 114:269-278

Rodrigues FHA, Fajardo AR, Ricardo NMPS et al (2012) Chitosan-graft-poly(acrylic acid)/rice husk ash based superabsorbent hydrogels composite: preparation and characterization. J Polym Res 19(12):1

Samir MASA, Alloin F, Dufresne A (2005) Review of recent research into cellulosic whiskers, their properties and their application in nanocomposite field. Biomacromolecules 6(2):612-626

Spagnol C, Rodrigues FHA, Pereira AGB et al (2012) Superabsorbent hydrogels nanocomposites based on starchpoly(sodium acrylate) matrix filled with cellulose nanowhiskers. Cellulose 19(4):1225-1237

Sun JY, Zhao X, Illeperuma WR et al (2012) Highly stretchable and tough hydrogels. Nature 489(7414):133-136

Sung Y, Kim TH, Lee B (2016) Syntheses of carboxymethylcellulose/graphene nanocomposite superabsorbent hydrogels with improved gel properties using electron beam radiation. Macromol Res 24(2):143-151

Tang L, Yang Y, Bai T et al (2011) Robust MeOMA/vinyl-4,6diamino-1,3,5-triazine copolymer hydrogels-mediated reverse gene transfection and thermo-induced cell detachment. Biomaterials 32(7):1943-1949

Xin L, Xu S, Wang J et al (2009) Structure and characterization of amphoteric semi-IPN hydrogels based on cationic starch. Carbohydr Polym 75(4):688-693

Yadav M, Rhee KY (2012) Superabsorbent nanocomposite (alginate-g-PAMPS/MMT): synthesis, characterization and swelling behavior. Carbohydr Polym 90(1):165-173

Zhang J, Wang Q, Wang A (2007) Synthesis and characterization of chitosan-poly(acrylic acid)/attapulgite superabsorbent composites. Carbohydr Polym 68(2):367-374

Zhang M, Cheng Z, Zhao T et al (2014) Synthesis, characterization, and swelling behaviors of salt-sensitive maize branpoly(acrylic acid) superabsorbent hydrogels. J Agric Food Chem 62(35):8867-8874

Zhang T, Cheng Q, Ye D et al (2017) Tunicate cellulose nanocrystals reinforced nanocomposite hydrogels comprised by hybrid cross-linked networks. Carbohydr Polym 169:139-148

Publisher's Note Springer Nature remains neutral with regard to jurisdictional claims in published maps and institutional affiliations. 